\title{
Structural dynamics of human FACT protein complex: electron microscopy analysis
}

Olesya Volokh $^{1}$, Anastasiia Sivkina ${ }^{2}$, Maria Karlova $^{1}$, Elena Kotova ${ }^{3}$, Vasily Studitsky ${ }^{4}$ and Olga Sokolova $^{1}$

${ }^{1}$ Moscow Lomonosov University, United States, ${ }^{2}$ Faculty of Biology, Lomonosov Moscow State University, 119234 Moscow, Russia., United States, ${ }^{3}$ Fox Chase Cancer Center, United States, ${ }^{4}$ Fox Chase Cancer Center, Philadelphia, PA 19111, USA., Philadelphia, Pennsylvania, United States

FACT is a multifunctional histone chaperone involved in transcription, DNA replication and repair [1]. Human FACT is a heterodimer of SPT16/SSRP1 subunits (Fig. 1A) that moderately stabilizes nucleosomes [2]. hFACT is being used as a target for anticancer drugs curaxins, which cause FACT redistribution and trapping in chromatin of cancer cells [3]. During that process, curaxins induce a large scale, FACT-dependent, ATPindependent and reversible nucleosome unfolding that modulates the accessibility of nucleosomal DNA via an 'all-or-none' mechanism, and occurs without apparent loss of histones [3] hFACT can also unfold nucleosomes in the presence of Nhp6 protein [4]. The nucleosome-unfolding activity is an important function of hFACT in vivo, but the detailed mechanism of this process is unknown. Recently, the structure of hFACT in complex with partially assembled subnucleosomes was studied using cryo-EM [5, 6]. In the absence of nucleosomes, a large DNA-binding surface of FACT is protected by C-terminal domains (CTD) of both of its subunits, and this inhibition is released by its interaction with $\mathrm{H} 2 \mathrm{~A}-\mathrm{H} 2 \mathrm{~B}$ dimer, allowing FACT-H2A-H2B complex to bind to $\mathrm{H} 3 / \mathrm{H} 4$ tetrasome $[5,6]$. However, the structure and dynamics of free hFACT has not been studied in detail yet. Here we studied human FACT using single particle electron microscopy. We evaluated the set of four different conformational states and proposed the hypothesis of human FACT structural dynamics. This human FACT conformational flexibility can be the starting point in understanding of initiation of structural interaction between FACT and nucleosome as well as an important step for evaluation of more complex FACT dynamics. We analyzed hSpt16/SSRP1 using JEOL 2100 TEM operated at $200 \mathrm{kV}$ at low-dose conditions. Micrographs were captured by the Gatan Ultrascan camera with $25 \mathrm{k}$ magnification, with $4.1 \AA$ pixel size. EM images preprocessing and single particles collection were performed in EMAN2.3, followed by 2D-particles analysis in RELION2.0. Final 2D-classes included $\sim 70 \mathrm{k}$ good single particles images. The free hFACT is a dynamic structure with three or four domains visible, depending on the conformation of the complex (Fig. 1B). Based on 2D-classess data analysis we evaluated four states of hFACT reflecting its conformational flexibility: the "closed" complex is characterized by four compact domains, positioned close to each other; "intermediates 1 and 2" are represented by classes with three domains forming a compact structure or containing a disordered fourth domain, and the "open" complex, represented by three domains forming a nearly linear structure. The distribution of single particles between these states (Fig. 2) suggests that the "closed" and "open" states are present in similar amounts. Based on the previously published structure of FACT-subnucleosome complex [5, 6], a mechanism of conformational flexibility of human FACT has been proposed (Fig. 1C). It has been shown that SPT16 and SSRP1 DDs are connected with the other FACT domains through flexible joints [5, 6]. In the "closed" conformation of FACT the DNA-binding surface of FACT is covered by its two C-terminal MD domains, forming a sharp angle between them (Fig. 1C). The N-terminal domain (NTD) of SPT16 was not resolved previously [6], but it is the best candidate for the forth domain visible in our "closed" conformation, based on its dimensions and the longest linker length. During conversion to the "open" complexes the NTD is moving away from the other subunits, forming the intermediates 1 and 2 where the NTD is weakly resolved or not resolved, respectively. In the intermediates the DD and MD domains maintain a compact structure with DNA-binding sites protected. Finally, in the "open" state the SPT16/SSRP1 MD domains are arranged in a nearly linear structure, resulting in unmasking of the DNA-binding sites of FACT and making them available for interaction with the nucleosome. 




Figure 1. Figure 1. Structural analysis of human FACT: electron microscopy. (A) Schematic of human FACT domain structure. NTD - N-terminal domain; DD - dimerization domain; MD - middle domain; CTD - Cterminal domain; IDD - intrinsically disordered domain. The sequential numbers of amino acid residue and linkers lengths are indicated. (B) 2D class-averages of hFACT. Left to right: closed, intermediate 1 and 2, open states; bar $-10 \mathrm{~nm}$. Below each class average a colored view is shown. Domain colors are same as in (A). (C) Schematic of domain movement during conformational changes in hFACT. Domain colors are same as in (A). Dotted lines demonstrate the changing from acute to obtuse angles between SPT16 and SSRP1 MDs upon opening of the hFACT. 


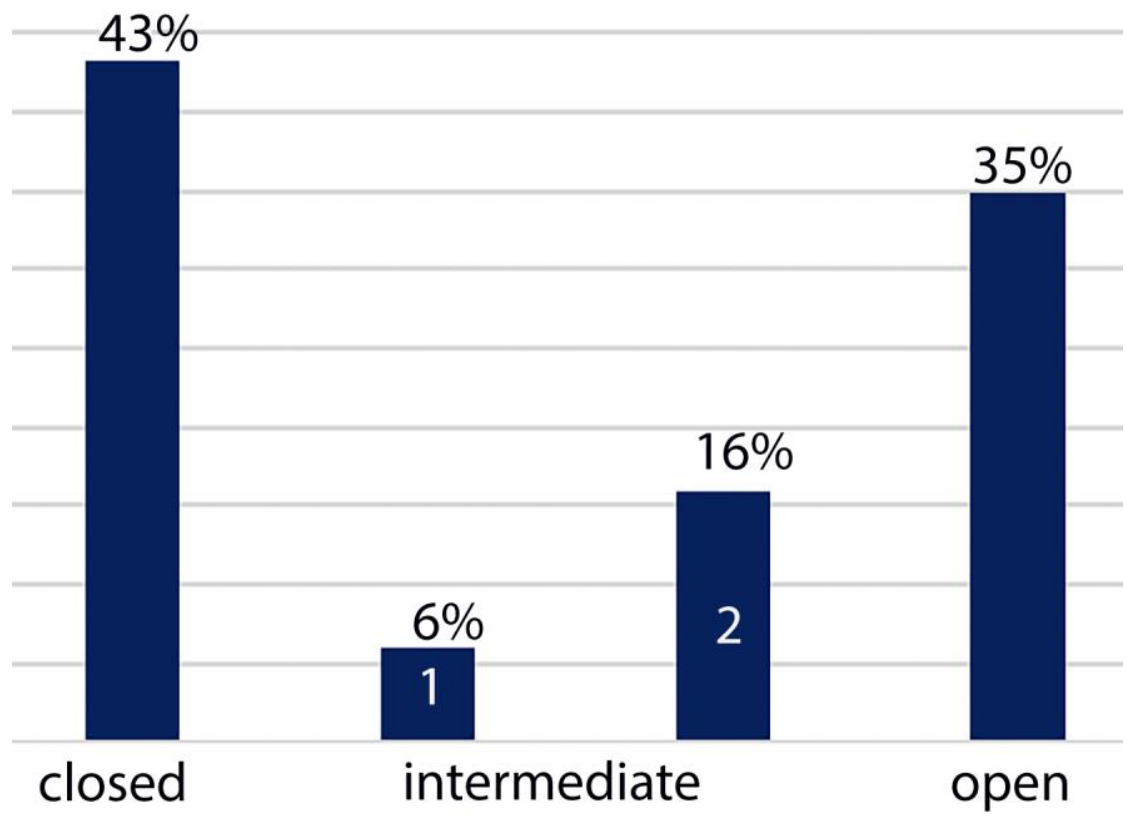

Figure 2. Figure 2.The distribution of "closed", "intermediate" and "open" states of human FACT.

\section{References}

1. Gurova, K., et al., Structure and function of the histone chaperone FACT - Resolving FACTual issues. Biochimica et Biophysica Acta (BBA) - Gene Regulatory Mechanisms, 2018. 1861(9): p. 892-904.

2. Valieva, M.E., et al., Stabilization of Nucleosomes by Histone Tails and by FACT Revealed by spFRET Microscopy. Cancers (Basel), 2017. 9(1).

3. Chang, H.W., et al., Mechanism of FACT removal from transcribed genes by anticancer drugs curaxins. Sci Adv, 2018. 4(11): p. eaav2131.

4. McCullough, L.L., et al., Functional roles of the DNA-binding HMGB domain in the histone chaperone FACT in nucleosome reorganization. J Biol Chem, 2018. 293(16): p. 6121-6133.

5. Mayanagi, K., et al., Structural visualization of key steps in nucleosome reorganization by human FACT. Sci Rep, 2019. 9(1): p. 10183.

6. Liu, Y., et al., FACT caught in the act of manipulating the nucleosome. Nature, 2020. 577(7790): p. 426431.

7. This work was supported by the Russian Science Foundation (\#19-74-30003). Electron microscopy was performed on the Unique equipment setup "3D-EMC” of Moscow State University, Department of Biology. 